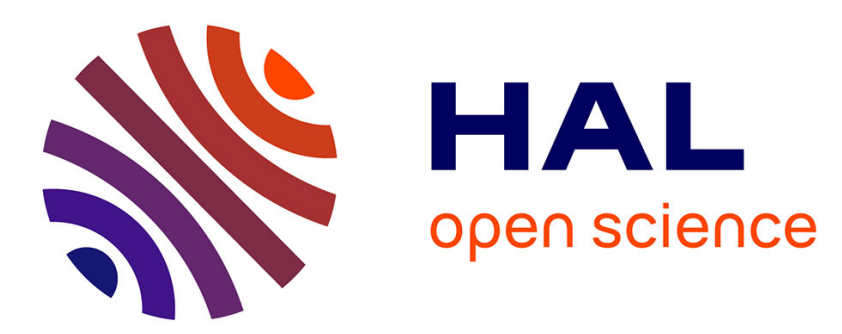

\title{
Adaptive synchronization of unknown heterogeneous agents: an adaptive virtual model reference approach
} Simone Baldi, Paolo Frasca

\section{To cite this version:}

Simone Baldi, Paolo Frasca. Adaptive synchronization of unknown heterogeneous agents: an adaptive virtual model reference approach. Journal of The Franklin Institute, 2019, 356 (2), pp.935-955. 10.1016/j.jfranklin.2018.01.022 . hal-01702085

\section{HAL Id: hal-01702085 \\ https://hal.science/hal-01702085}

Submitted on 6 Feb 2018

HAL is a multi-disciplinary open access archive for the deposit and dissemination of scientific research documents, whether they are published or not. The documents may come from teaching and research institutions in France or abroad, or from public or private research centers.
L'archive ouverte pluridisciplinaire HAL, est destinée au dépôt et à la diffusion de documents scientifiques de niveau recherche, publiés ou non, émanant des établissements d'enseignement et de recherche français ou étrangers, des laboratoires publics ou privés. 


\title{
Adaptive synchronization of unknown heterogeneous agents: an adaptive virtual model reference approach
}

\author{
Simone Baldi ${ }^{a}$, Paolo Frasca ${ }^{b}$ \\ ${ }^{a}$ Delft Center for Systems and Control, Delft University of Technology, Delft 2628CD, The Netherlands \\ ${ }^{b}$ Univ. Grenoble Alpes, CNRS, Inria, Grenoble INP* , GIPSA-Lab, F-38000 Grenoble, France
}

\begin{abstract}
This work deals with state synchronization of heterogeneous linear agents with unknown dynamics. The problem is solved by formulating the synchronization problem as a special model reference adaptive control where each agent tries to converge to the model defined by its neighbors. For those agents that do not know the reference signal that drives the flock, a fictitious reference is estimated in place of the actual one: the estimation of such reference is distributed and requires measurements from neighbors. By using a matching condition assumption, which is imposed so that the agents can converge to the same behavior, the fictitious reference estimation leads to adaptive laws for the feedback and coupling gains arising from distributed matching conditions. In addition, the coupling connection is not scalar, but possibly vector-valued. The proposed approach is applicable to heterogeneous agents with arbitrarily large matched uncertainties. A Lyapunov-based approach is derived to show analytically asymptotic convergence of the synchronization error: robustification in the presence of bounded errors or unknown (constant) leader input is also discussed. Finally, a motivational example is presented in the context of Cooperative Adaptive Cruise Control and numerical examples are provided to demonstrate the effectiveness of the proposed method.
\end{abstract}

Keywords:

Adaptive synchronization, heterogeneous uncertain agents, adaptive control, virtual reference model.

\section{Introduction}

In recent years, the cooperative control of multiagent systems has received the attention of many scientific communities, due to its impact in crucial areas such as formation flying, unmanned aerial vehicles, smart traffic, computer networks and many more $[1,2,3,4,5,6]$. An important problem in cooperative control is to achieve in a distributed way (i.e. using local information) a common behavior for the entire network: this is the so-called synchronization problem [7, 8]. A topic that is closely related to synchronization of multiagent systems is consensus $[9,10,11,12]$ : the connections between consensus and synchronization has been recently highlighted in [13, 14]. In the following we will discuss some literature from both synchronization and consensus areas.

Historically, one of the main drive for studying synchronization is flocking and swarming behaviors [15]. Flocking and swarming are the behaviors exhibited when a group of living beings such as birds, fish, bacteria, and insects are interacting with each other to accomplish a task (e.g. foraging, flying or shoaling) [16]: motivated by this interest, research has focused on synchronization without a leader (also called leaderless consensus), and on synchronization with an active leader having knowledge of a reference signal (also called leader-following consensus) [17, 18]. An important achievement in these fields was achieving synchronization or consensus for multi-agent systems with general linear dynamics without the knowledge of the eigenvalues of the Laplacian matrix, or of the smallest eigenvalue

\footnotetext{
${ }^{*}$ Institute of Engineering Univ. Grenoble Alpes

Email addresses: s.baldi@tudelft.nl (Simone Baldi), paolo.frascaegipsa-lab.fr (Paolo Frasca)

${ }^{1}$ The first author has been partially supported by the European Commission FP7-ICT-2013.3.4, Advanced computing, embedded and control systems, under contract \#611538 (LOCAL4GLOBAL)
} 
different than zero (the so-called algebraic connectivity). The knowledge of these eigenvalues is actually global information, because it requires the knowledge of the network topology. To overcome this limitation, distributed adaptive protocols have been developed, where, instead of having fixed coupling weights among agents, some scalar couplings are updated online $[19,20]$. Both consensus and synchronization are currently well-established research areas: however, some major problems still need to be addressed, two of the biggest ones being how to guarantee synchronization in the presence of (a) heterogeneous agents and (b) uncertain agents: note that these two instances may not coincide, since agents can be uncertain but homogeneous, as considered in [21, 22], or heterogeneous with no uncertainty $[23,24]$ or bounded/structural uncertainty $[25,26,27]$. In both instances one would like to delete the effect of heterogeneity or uncertainty via feedback gains: up to now, non-adaptive feedback (i.e. with fixed feedback gains) with possibly adaptive coupling weights is commonly employed. In other words, fixed feedback gains are designed based on a nominal agent [28], while only the coupling weights are updated online.

For cooperative control strategies to be effective in unstructured environments, teams of agents must be able to respond to unanticipated situations or changes in the environment that might occur while the cooperative task is carried out. To this purpose, it is of fundamental importance to integrate adaptive control methodologies in cooperative control and investigate whether it could be possible to update online in an adaptive way both the feedback gains and the coupling weights. Currently, only in the restrictive class of second-order Euler-Lagrangian agents, researchers have developed control architectures where both the feedback gains and the coupling weights are updated online [29, 30].

This work thus explores the direction of having fully adaptive synchronization approaches in both feedback and coupling weights arising from distributed matching conditions for heterogeneous agents with large uncertainties. By looking at the synchronization problem of uncertain heterogeneous agents as a particular model reference adaptive control where each agent tries to converge to the model defined by its neighbors, we are able to derive a set of 'feedback matching conditions' (that link the agent to the reference model) and a set of 'coupling matching conditions' (that link the agent to its neighbors). These conditions give rise, for those agents that cannot access the reference signal, to a fictitious reference in place of the actual one. Due to uncertainty, the fictitious reference has to be estimated and requires the agent to observe the relative state but also the control input of its neighbors.

The main contributions of this work are: to propose adaptive synchronization laws accounting for online adaptation of both feedback gains and coupling weights; and to consider heterogeneous agents with arbitrarily large matched uncertainties. A Lyapunov-based approach is derived to show analytically that the synchronization error converges asymptotically to zero in the ideal noiseless case, or is bounded in case the neighbors' input is evaluated with some misjudgement error. Finally, an estimation architecture is proposed in case the neighbors' input cannot be evaluated. Simulations demonstrate the effectiveness of the proposed approach.

At the end of this introductory section, we would like to open a parenthesis on the role of knowing the control input to achieve synchronization tasks: while it is well accepted that relative measurements are used by flocks in nature (e.g. relative position/velocity), it is not clear whether each component of the flock also uses the information of the input of its neighbors (e.g. how neighbors flap their wings or fins) [15]. On the other hand, humans may indeed use some input knowledge for better synchronization: cyclists riding in a peloton in races such as the Tour De France and the Giro d'Italia make adjustments according to others in the group in order to keep the formation [31]: whether such adjustments are facilitated by the observation of the input applied by neighboring cyclists (handlebar and body movements) is debated, but the cycling etiquette suggests that such information is important [32]. Surely, in many technological domains, the knowledge the inputs applied by neighbors is effectively used: e.g. the centre brake light high-mounted in vehicles is requested by law in most countries with the aim to reduce rear-end crashes (i.e. to improve synchronization). Going one step further, in Cooperative Adaptive Cruise Control (CACC) communication of the (input) acceleration from the preceding to the following vehicles is used to maintain a safe inter-vehicle distance [33]: a similar architecture is also currently studied for platoons that need to control not only the longitudinal, but also the lateral dynamics by communicating steering commands [34]. With these examples in mind, we close the parenthesis by noticing that CACC could be a direct engineering application of the proposed synchronization algorithm.

The rest of the paper is organized as follows: Section 2 introduces some preliminary concepts useful to understand the novel adaptation idea. Section 3 extends these concepts to the leader-follower synchronization setting, and develops appropriate adaptation mechanisms to have the synchronization error converging to zero. Section 4 includes some robustness mechanisms to account for bounded errors and/or unknown input of the leader. Section 5 discusses the CACC application in view of the proposed approach and Section 6 presents some simulations to demonstrate the theoretical findings. Section 7 concludes the paper. 
Notation: The notation in this paper is standard. Matrices are denoted by capital letters, e.g. $X$, and vectors by small letters, e.g. $x$. The transpose of a matrix or of a vector is indicated with $X^{\prime}$ and $x^{\prime}$ respectively. The Euclidean norm of a vector $x=\left(x_{1}, x_{2}, \ldots, x_{n}\right)$ is $\|x\|=\sqrt{\sum_{i=1}^{n}\left|x_{i}\right|^{2}}$. The trace of a square matrix $X$ is $\operatorname{tr}[X]$. A vector signal $x \in \mathbb{R}^{n}$ is said to belong to $\mathscr{L}_{2}$ class $\left(x \in \mathscr{L}_{2}\right)$, if $\int_{0}^{t}\|x(\tau)\|^{2} \mathrm{~d} \tau<\infty, \forall t \geq 0$. A vector signal $x \in \mathbb{R}^{n}$ is said to belong to $\mathscr{L}_{\infty}$ class $\left(x \in \mathscr{L}_{\infty}\right)$, if $\max _{t \geq 0}\|x(t)\|<\infty, \forall t \geq 0$. A directed graph (digraph) is indicated with the pair $(\mathscr{N}, \mathscr{E})$, where $\mathscr{N}$ is a finite nonempty set of nodes, and $\mathscr{E} \in \mathscr{N} \times \mathscr{N}$ is a set of ordered pair of nodes, called edges. The adjacency matrix $\mathscr{A}=\left[a_{i j}\right]$ of an unweighted digraph is defined as $a_{i i}=0$ and $a_{i j}=1$ if $(j, i) \in \mathscr{E}$, where $i \neq j$. The Laplacian matrix of the unweighted digraph is defined as $L=\left[l_{i j}\right]$, where $l_{i i}=\sum_{j} a_{i j}$ and $l_{i j}=-a_{i j}$, where $i \neq j$.

\section{Preliminary results}

In order to facilitate the presentation of the main results, we first formulate the proposed synchronization problem for two agents. Let us consider two agents, denoted with 1 and 2, with dynamics

$$
\begin{aligned}
& \dot{x}_{1}=A_{1} x_{1}+b_{1} u_{1} \\
& \dot{x}_{2}=A_{2} x_{2}+b_{2} u_{2}
\end{aligned}
$$

where $x_{1}, x_{2} \in \mathbb{R}^{n}$ is the state, $u_{1}, u_{2} \in \mathbb{R}$ is the input, and $A_{1}, A_{2}$ and $b_{1}, b_{2}$ are unknown matrices of appropriate dimensions, with possibly $A_{1} \neq A_{2}$ and $b_{1} \neq b_{2}$ (heterogeneous unknown agents). We assume a directed connection from 1 to 2, i.e. the digraph is described by $\mathscr{N}=\{1,2\}, \mathscr{E}=\{(1,2)\}$. With this directed connection, agent 2 can observe measurements from agent 1 , but not viceversa. Assume that the purpose of agent 1 is to follow a reference model

$$
\dot{x}_{m}=A_{m} x_{m}+b_{m} r
$$

where $x_{m} \in \mathbb{R}^{n}$ is the state of the reference model, $r \in \mathbb{R}$ is the reference input of the reference model, and $A_{m}$ and $b_{m}$ are known matrices of appropriate dimensions, with $A_{m}$ being Hurwitz so as to have a bounded state trajectory $x_{m}$ for bounded $r$. The synchronization task is achieved when $x_{1} \rightarrow x_{m}$ for $t \rightarrow \infty$. The reference model (2) can also be interpreted as agent 0 , whose signals are known to agent 1 only: the purpose of agent 2 is to synchronize to agent 1 , i.e. $x_{2} \rightarrow x_{1}$ for $t \rightarrow \infty$. It is clear that if both synchronization tasks are achieved, we have $x_{2} \rightarrow x_{m}$ for $t \rightarrow \infty$.

Being the system matrices in (1) unknown, the synchronization task has to be achieved in an adaptive fashion. In order to have a well-posed adaptive control problem, the following assumptions should be verified.

Assumption 1. There exist constant vectors $k_{1}^{*}, k_{2}^{*}$ and scalars $l_{1}^{*}, l_{2}^{*}$ such that

$$
\begin{array}{ll}
A_{m}=A_{1}+b_{1} k_{1}^{* \prime}, & b_{m}=b_{1} l_{1}^{*} \\
A_{m}=A_{2}+b_{2} k_{2}^{* \prime}, & b_{m}=b_{2} l_{2}^{*} .
\end{array}
$$

Assumption 2. The signs of $l_{1}^{*}, l_{2}^{*}$ are known.

Remark 1. Both Assumptions 1 and 2 are mutuated from model reference adaptive control (single-input singlereference case): in particular, Assumption 1 is required for the existence of a closed-loop system, for both agents 1 and 2 that matches the reference model (2): for this reason, let us refer to (3) as 'feedback matching conditions'. Assumption 2 basically amounts to the condition, classical in adaptive control, of knowing the sign of the input vector field.

Remark 2. Often synchronization is sought for classes of reference signals generated by an exogenous system [24]: in this work we look at synchronization tasks for arbitrary reference signals, which requires the input dimension to be greater or equal to the reference dimension: for simplicity, we look at the square case (same number of inputs and references), and in particular at the single-input single-reference case. For the multiple-input multiple-reference case, the interested reader is referred to the comprehensive survey [35], where it is explained how in place of Assumption 2 one needs to assume the existence of a known matrix $S$ such that $\Gamma=l_{1}^{*} S$ (where $l_{1}^{*}$ is now a matrix) is symmetric and 
positive definite. Since the extension to multiple inputs and multiple references introduces technicalities that might hinder the core contribution of this work, in order to keep the presentation simple, let us carry out the design for the single-input single-reference case.

The following result is a consequence of Assumption 1.

Proposition 1. There exist a constant vector $k_{21}^{*}$ and a scalar $l_{21}^{*}$ such that

$$
A_{1}=A_{2}+b_{2} k_{21}^{* \prime}, \quad b_{1}=b_{2} l_{21}^{*} .
$$

The condition (4), proven below, implies that agent 2 can match the model of agent 1 via appropriate gains: for this reason, let us refer to (4) as 'coupling matching conditions'.

Proof. From (3) we find

$$
\begin{aligned}
b_{1} & =b_{2} \frac{l_{2}^{*}}{l_{1}^{*}} \\
A_{1}-A_{2} & =b_{2} k_{2}^{* \prime}-b_{1} k_{1}^{* \prime} \\
A_{1}-A_{2} & =b_{2}\left[k_{2}^{* \prime}-\frac{l_{2}^{*}}{l_{1}^{*}} k_{1}^{* \prime}\right]
\end{aligned}
$$

which is in the form of (4) with $k_{21}^{* \prime}=k_{2}^{* \prime}-\frac{l_{2}^{*}}{l_{1}^{*}} k_{1}^{* \prime}$ and $l_{21}^{*}=\frac{l_{2}^{*}}{l_{1}^{*}}$.

Using classical model reference adaptive control tools [36, Chap. 4], it is well known that agent 1 can synchronize to the reference model (2) via the controller

$$
u_{1}(t)=k_{1}^{\prime}(t) x_{1}(t)+l_{1}(t) r(t)
$$

and the adaptive laws

$$
\begin{aligned}
\dot{k}_{1}^{\prime}(t) & =-\operatorname{sgn}\left(l_{1}^{*}\right) \gamma b_{m}^{\prime} P\left(x_{1}(t)-x_{m}(t)\right) x_{1}(t)^{\prime} \\
\dot{l}_{1}(t) & =-\operatorname{sgn}\left(l_{1}^{*}\right) \gamma b_{m}^{\prime} P\left(x_{1}(t)-x_{m}(t)\right) r(t)
\end{aligned}
$$

where the scalar $\gamma>0$ is the adaptive gain, and $P$ is a positive definite matrix satisfying

$$
P A_{m}+A_{m}^{\prime} P=-Q, \quad Q>0
$$

and $k_{1}, l_{1}$ are the estimates of $k_{1}^{*}, l_{1}^{*}$ respectively. For completeness, in the following we recall how the adaptation laws in (7) can be derived via the dynamics of the error $e_{1}=x_{1}-x_{m}$

$$
\dot{e}_{1}(t)=A_{m} e_{1}(t)+b_{1}\left(\tilde{k}_{1}^{\prime}(t) x_{1}(t)+\tilde{l}_{1}(t) r(t)\right)
$$

with $\tilde{k}_{1}=k_{1}-k_{1}^{*}, \tilde{l}_{1}=l_{1}-l_{1}^{*}$. Using the Lyapunov function (time index $t$ is omitted for compactness)

$$
V_{1}\left(e_{1}, \tilde{k}_{1}, \tilde{l}_{1}\right)=e_{1}^{\prime} P e_{1}+\operatorname{tr}\left(\frac{\tilde{k}_{k}^{\prime} \tilde{k}_{i}}{\gamma\left|l_{i}^{*}\right|}\right)+\frac{\tilde{l}_{i}^{2}}{\gamma\left|l_{i}^{*}\right|}
$$

it is possible to verify

$$
\begin{aligned}
\dot{V}_{1}= & e_{1}^{\prime}\left(P A_{m}+A_{m}^{\prime} P\right) e_{1}+2 e_{1}^{\prime} P b_{i}\left(\tilde{k}_{1}^{\prime} x_{1}+\tilde{l}_{1} r\right)+2 \operatorname{tr}\left(\frac{\tilde{k}_{1}^{\prime} \gamma^{-1} \dot{\tilde{k}}_{1}}{\left|l_{1}^{*}\right|}\right)+2 \frac{\tilde{l}_{1} \gamma^{-1} \dot{\tilde{l}}_{1}}{\left|l_{1}^{*}\right|} \\
= & -e_{1}^{\prime} Q e_{1}+2\left(\operatorname{sgn}\left(l_{1}^{*}\right) b_{m}^{\prime} P\left(x_{1}-x_{m}\right) x_{1}^{\prime}+\gamma^{-1} \dot{\tilde{k}}_{1}^{\prime}\right) \frac{\tilde{k}_{1}}{\left|l_{1}^{*}\right|} \\
& \quad+2\left(\operatorname{sgn}\left(l_{1}^{*}\right) b_{m}^{\prime} P\left(x_{1}-x_{m}\right) r+\gamma^{-1} \dot{\tilde{l}}_{i}\right) \frac{\tilde{l}_{1}}{\left|l_{1}^{*}\right|} \\
= & -e_{1}^{\prime} Q e_{1}
\end{aligned}
$$


where we have used (7) and the property $a^{\prime} b=\operatorname{tr}\left(b^{\prime} a\right)$. Using standard Lyapunov arguments we can prove boundedness of all closed-loop signals and convergence of $e_{1}$ to 0 . In fact, since $V_{1}>0$ and $\dot{V}_{1} \leq 0$, it follows that $V_{1}(t)$ has a limit, i.e.,

$$
\lim _{t \rightarrow \infty} V_{1}\left(e_{1}(t), \tilde{k}_{1}(t), \tilde{l}_{1}(t)\right)=V_{\infty}<\infty
$$

and $V_{1}, e_{1}, \tilde{k}_{1}, \tilde{l}_{1} \in \mathscr{L}_{\infty}$. In addition, by integrating $\dot{V}_{1}$ it follows that

$$
\int_{0}^{\infty} e_{1}^{\prime}(\tau) Q e_{1}(\tau) \mathrm{d} \tau \leq V\left(e_{1}(0), \tilde{k}_{1}(0), \tilde{l}_{1}(0)\right)-V_{\infty}
$$

from which we establish that $e_{1} \in \mathscr{L}_{2}$. Finally, since $\dot{V}_{1}$ is uniformly continuous in time (this is satisfied because $\ddot{V}_{1}$ is finite), the Barbalat's lemma implies $\dot{V}_{1} \rightarrow 0$ as $t \rightarrow \infty$ and hence $e_{1} \rightarrow 0$.

The purpose is now to find an adaptive law that can synchronize agent 2 to agent 1 without using the reference input $r$. We observe that this is possible via the controller

$$
u_{2}(t)=k_{21}^{\prime}(t) x_{1}(t)+k_{2}^{\prime}(t)\left(x_{2}(t)-x_{1}(t)\right)+l_{21}(t) u_{1}(t)
$$

and the adaptive laws

$$
\begin{aligned}
\dot{k}_{21}^{\prime}(t) & =-\operatorname{sgn}\left(l_{2}^{*}\right) \gamma b_{m}^{\prime} P\left(x_{2}(t)-x_{1}(t)\right) x_{1}(t)^{\prime} \\
\dot{k}_{2}^{\prime}(t) & =-\operatorname{sgn}\left(l_{2}^{*}\right) \gamma b_{m}^{\prime} P\left(x_{2}(t)-x_{1}(t)\right)\left(x_{2}(t)-x_{1}(t)\right)^{\prime} \\
\dot{l}_{21}(t) & =-\operatorname{sgn}\left(l_{2}^{*}\right) \gamma b_{m}^{\prime} P\left(x_{2}(t)-x_{1}(t)\right) u_{1}(t)
\end{aligned}
$$

where $k_{21}, k_{2}, l_{21}$ are the estimates of $k_{21}^{*}, k_{2}^{*}, l_{21}^{*}$ respectively. It has to be noted that we do not need to estimate $l_{2}^{*}$, even though we need its sign to implement the adaptive law. Let us derive the adaptation law in (15) via the dynamics of the error $e_{21}=x_{2}-x_{1}$

$$
\dot{e}_{21}=A_{m} e_{21}+b_{2}\left(\tilde{k}_{21}^{\prime} x_{1}+\tilde{k}_{2}^{\prime} e_{21}+\tilde{l}_{21} u_{1}\right)
$$

with $\tilde{k}_{21}=k_{21}-k_{21}^{*}, \tilde{k}_{2}=k_{2}-k_{2}^{*}, \tilde{l}_{21}=l_{21}-l_{21}^{*}$. By taking the Lyapunov function

$$
V_{21}=e_{21}^{\prime} P e_{21}+\operatorname{tr}\left(\frac{\tilde{k}_{21}^{\prime} \tilde{k}_{21}}{\gamma\left|l_{2}^{*}\right|}\right)+\operatorname{tr}\left(\frac{\tilde{k}_{2}^{\prime} \tilde{k}_{2}}{\gamma\left|l_{2}^{*}\right|}\right)+\frac{\tilde{l}_{21}^{2}}{\gamma\left|l_{2}^{*}\right|}
$$

we can calculate the time derivative of (17) along (16)

$$
\begin{aligned}
\dot{V}_{21}= & e_{21}^{\prime}\left(P A_{m}+A_{m}^{\prime} P\right) e_{21}+2 e_{21}^{\prime} P b_{2}\left(\tilde{k}_{21}^{\prime} x_{i}+\tilde{k}_{2}^{\prime} e_{21}+\tilde{l}_{21} u_{1}\right) \\
& +2 \operatorname{tr}\left(\frac{\tilde{k}_{21}^{\prime} \gamma^{-1} \dot{\tilde{k}}_{21}}{\left|l_{2}^{*}\right|}\right)+2 \operatorname{tr}\left(\frac{\tilde{k}_{2}^{\prime} \gamma^{-1} \dot{\tilde{k}}_{2}}{\left|l_{2}^{*}\right|}\right)+2 \frac{\tilde{l}_{21}^{\prime} \gamma^{-1} \dot{\tilde{l}}_{21}}{\left|l_{2}^{*}\right|} \\
= & -e_{21}^{\prime} Q e_{21}+2\left(\operatorname{sgn}\left(l_{2}^{*}\right) b_{m}^{\prime} P e_{21} x_{1}^{\prime}+\gamma^{-1} \dot{\tilde{k}}_{21}^{\prime}\right) \frac{\tilde{k}_{21}^{\prime}}{\left|l_{2}^{*}\right|} \\
& \quad+2\left(\operatorname{sgn}\left(l_{2}^{*}\right) b_{m}^{\prime} P e_{21} e_{21}^{\prime}+\gamma^{-1} \dot{\tilde{k}}_{2}^{\prime}\right) \frac{\tilde{k}_{2}}{\left|l_{2}^{*}\right|}+2\left(\operatorname{sgn}\left(l_{2}^{*}\right) b_{m}^{\prime} P e_{21} u_{1}+\gamma^{-1} \dot{\tilde{l}}_{21}\right) \frac{\tilde{l}_{21}^{\prime}}{\left|l_{2}^{*}\right|} \\
= & -e_{21}^{\prime} Q e_{21}
\end{aligned}
$$

where we have used (15). Using standard Lyapunov arguments we can prove boundedness of all closed-loop signals and convergence of $e_{21}$ to 0 . In fact, since $V_{21}>0$ and $\dot{V}_{21} \leq 0$, it follows that $V_{21}(t)$ has a limit, i.e.,

$$
\lim _{t \rightarrow \infty} V_{21}\left(e_{21}(t), \tilde{k}_{21}(t), \tilde{k}_{2}(t), \tilde{l}_{21}(t)\right)=V_{\infty}<\infty
$$

and $V_{21}, e_{21}, \tilde{k}_{21}, \tilde{k}_{2}, \tilde{l}_{21} \in \mathscr{L}_{\infty}$. In addition, by integrating $\dot{V}_{21}$ it follows that

$$
\int_{0}^{\infty} e_{21}^{\prime}(\tau) Q e_{21}(\tau) \mathrm{d} \tau \leq V\left(e_{21}(0), \tilde{k}_{21}(0), \tilde{k}_{2}(0), \tilde{l}_{21}(0)\right)-V_{\infty}
$$

from which we establish that $e_{21} \in \mathscr{L}_{2}$. Finally, since $\dot{V}_{21}$ is uniformly continuous in time (this is satisfied because $\ddot{V}_{21}$ is finite), the Barbalat's lemma implies $\dot{V}_{21} \rightarrow 0$ as $t \rightarrow \infty$ and hence $e_{21} \rightarrow 0$.

The following result, whose proof has been constructed in the previous steps, can be stated. 
Proposition 2. Consider the unknown heterogeneous linear systems (1), with reference model (2), controllers (6) and (14) and update laws (7) and (15). Then all closed-loop signals are bounded and $e_{1}=x_{1}-x_{m}, e_{21}=x_{2}-x_{1}$ converge asymptotically to zero.

Some remarks follow:

Remark 3. The following structure can be recognized in controller (14): the adaptive laws try to match agent 2 to the reference model (by estimating $k_{2}^{*}$ ) and at the same time to match agent 2 to agent 1 (by estimating $k_{21}^{*}$ and $l_{21}^{*}$ ). Then, it is reasonable to refer to $k_{1}, l_{1}, k_{2}$ and $l_{2}$ as to the feedback gains of agents 1 and 2 , and to $k_{21}$ and $l_{21}$ as to the coupling gains of agent 2 with respect to agent 1 . Also note that agent 2 synchronizes to agent 1 without using the knowledge of the reference signal $r$ (which is known to agent 1 only): however, $u_{1}$ must be accessible to agent 2 . In order to understand how the knowledge of the reference can be avoided, let us define from (6) and (14) the following ideal controls

$$
\begin{aligned}
u_{1}^{*}(t) & =k_{1}^{* \prime} x_{1}(t)+l_{1}^{*} r(t) \\
u_{2}^{*}(t) & =k_{21}^{* \prime} x_{1}(t)+k_{2}^{* \prime}\left(x_{2}(t)-x_{1}(t)\right)+l_{21}^{*} u_{1}(t) \\
& =k_{2}^{* \prime} x_{2}(t)+l_{2}^{*}\left[\frac{k_{21}^{* \prime}}{l_{2}^{*}} x_{1}(t)-\frac{k_{2}^{* \prime}}{l_{2}^{*}} x_{1}(t)+\frac{l_{21}^{*}}{l_{2}^{*}} u_{1}(t)\right] \\
& =k_{2}^{* \prime} x_{2}(t)+l_{2}^{*}\left[-\frac{k_{1}^{* \prime}}{l_{1}^{*}} x_{1}(t)+\frac{1}{l_{1}^{*}} u_{1}(t)\right]
\end{aligned}
$$

from which we notice that the term inside the square parenthesis in (21) plays the role of a virtual reference for agent 2. We also note that if the agents where homogeneous, one would have $k_{21}^{*}=0$ and $l_{21}^{*}=1$, and the ideal input would simplify to

$$
u_{2}^{*}(t)=k_{2}^{* \prime}\left(x_{2}(t)-x_{1}(t)\right)+u_{1}(t) .
$$

Remark 4. The proposed approach does not require the unknown agents to be homogeneous: the agents (1) can be heterogeneous, but they have to be able to match a certain reference model $\left(A_{m}, b_{m}\right)$ via appropriate control gains. Note that, as common in adaptive control results, convergence of $k_{1}, k_{2}, k_{21}, l_{1}, l_{21}$ to $k_{1}^{*}, k_{2}^{*}, k_{21}^{*}, l_{1}^{*}, l_{21}^{*}$ is neither guaranteed, nor necessary for the synchronization error to converge to zero [37, Chap. 6]. Convergence to the true parameters will occur if the reference signal $r$ is persistently exciting.

We close this section by noticing that it is not difficult to verify that the synchronization result can be achieved also in case agent 1 has multiple followers, each one trying to synchronize to its behavior. Similarly, the same reasoning of Proposition 2 can be applied to any network with a directed tree topology in which each leaf has one parent and the root node has access to the reference signal $r$.

\section{Adaptive leader-follower synchronization}

Before giving the main result, it is functional to deal with the case in which a follower (called agent 3 ) tries to synchronize to two parent neighbors (called agents 1 and 2). We assume a directed connection from 1 to 3 and from 2 to 3 , i.e. the digraph is described by $\mathscr{N}=\{1,2,3\}, \mathscr{E}=\{(1,3),(2,3)\}$. In addition, let us consider for simplicity an unweighted digraph, i.e. $a_{13}=a_{23}=1$.

In order to synchronize agent 3 to agents 1 and 2, let us define the dynamics of the error $e_{31}=x_{3}-x_{1}$ and $e_{32}=x_{3}-x_{2}$

$$
\begin{aligned}
& \dot{e}_{31}=A_{m} e_{31}+b_{3}\left(u_{3}-k_{31}^{* \prime} x_{1}-k_{3}^{* \prime} e_{31}-l_{31}^{*} u_{1}\right) \\
& \dot{e}_{32}=A_{m} e_{32}+b_{3}\left(u_{3}-k_{32}^{* 1} x_{2}-k_{3}^{* \prime} e_{32}-l_{32}^{*} u_{2}\right)
\end{aligned}
$$

which, by considering the sum of the aforementioned dynamics $\left(e_{31}+e_{32}\right)$, leads us to the controller

$$
u_{3}(t)=k_{31}^{\prime}(t) \frac{x_{1}(t)}{2}+k_{32}^{\prime}(t) \frac{x_{2}(t)}{2}+k_{3}^{\prime}(t) \frac{e_{31}(t)+e_{32}(t)}{2}+l_{31}(t) \frac{u_{1}(t)}{2}+l_{32}(t) \frac{u_{2}(t)}{2}
$$


and the adaptive laws

$$
\begin{aligned}
\dot{k}_{31}^{\prime}(t) & =-\operatorname{sgn}\left(l_{3}^{*}\right) \gamma b_{m}^{\prime} P\left(e_{31}(t)+e_{32}(t)\right) x_{1}^{\prime}(t) \\
\dot{k}_{32}^{\prime}(t) & =-\operatorname{sgn}\left(l_{3}^{*}\right) \gamma b_{m}^{\prime} P\left(e_{31}(t)+e_{32}(t)\right) x_{2}^{\prime}(t) \\
\dot{k}_{3}^{\prime}(t) & =-\operatorname{sgn}\left(l_{3}^{*}\right) \gamma b_{m}^{\prime} P\left(e_{31}(t)+e_{32}(t)\right)\left(e_{31}(t)+e_{32}(t)\right)^{\prime} \\
\dot{l}_{31}(t) & =-\operatorname{sgn}\left(l_{3}^{*}\right) \gamma b_{m}^{\prime} P\left(e_{31}(t)+e_{32}(t)\right) u_{1}(t) \\
\dot{l}_{32}(t) & =-\operatorname{sgn}\left(l_{3}^{*}\right) \gamma b_{m}^{\prime} P\left(e_{31}(t)+e_{32}(t)\right) u_{2}(t)
\end{aligned}
$$

where $k_{31}, k_{32}, k_{3}, l_{31}, l_{32}$ are the estimates of $k_{31}^{*}, k_{32}^{*}, k_{3}^{*}, l_{31}^{*}, l_{32}^{*}$ respectively. Let us derive the adaptation law in (25) via the dynamics of the error $e_{321}=e_{31}+e_{32}$

$$
\dot{e}_{321}=A_{m} e_{321}+2 b_{3}\left(\tilde{k}_{31}^{\prime} \frac{x_{1}}{2}+\tilde{k}_{32}^{\prime} \frac{x_{2}}{2}+\tilde{k}_{3}^{\prime} \frac{e_{321}}{2}+\tilde{l}_{31} \frac{u_{1}(t)}{2}+\tilde{l}_{32} \frac{u_{2}(t)}{2}\right)
$$

By taking the Lyapunov function

$$
V_{321}=e_{321}^{\prime} P e_{321}+\operatorname{tr}\left(\frac{\tilde{k}_{31}^{\prime} \tilde{k}_{31}}{\gamma\left|l_{3}^{*}\right|}\right)+\operatorname{tr}\left(\frac{\tilde{k}_{32}^{\prime} \tilde{k}_{32}}{\gamma\left|l_{3}^{*}\right|}\right)+\operatorname{tr}\left(\frac{\tilde{k}_{3}^{\prime} \tilde{k}_{3}}{\gamma\left|l_{3}^{*}\right|}\right)+\frac{\tilde{l}_{31}^{2}}{\gamma\left|l_{3}^{*}\right|}+\frac{\tilde{l}_{32}^{2}}{\gamma\left|l_{3}^{*}\right|}
$$

it is possible to verify

$$
\begin{aligned}
\dot{V}_{321}= & e_{321}^{\prime}\left(P A_{m}+A_{m}^{\prime} P\right) e_{321}+2 e_{321}^{\prime} P b_{3}\left(\tilde{k}_{31}^{\prime} x_{1}+\tilde{k}_{32}^{\prime} x_{2}+\tilde{k}_{3}^{\prime} e_{321}+\tilde{l}_{31} u_{1}(t)+\tilde{l}_{32} u_{2}(t)\right) \\
& +2 \operatorname{tr}\left(\frac{\tilde{k}_{31}^{\prime} \gamma^{-1} \dot{\tilde{k}}_{31}}{\left|l_{3}^{*}\right|}\right)+2 \operatorname{tr}\left(\frac{\tilde{k}_{32}^{\prime} \gamma^{-1} \dot{\tilde{k}}_{32}}{\left|l_{3}^{*}\right|}\right)+2 \operatorname{tr}\left(\frac{\tilde{k}_{3}^{\prime} \gamma^{-1} \dot{\tilde{k}}_{3}}{\left|l_{3}^{*}\right|}\right)+2 \frac{\tilde{l}_{31} \gamma^{-1} \dot{\tilde{l}}_{31}}{\left|l_{3}^{*}\right|}+2 \frac{\tilde{l}_{32} \gamma^{-1} \dot{\tilde{l}}_{32}}{\left|l_{3}^{*}\right|} \\
= & -e_{321}^{\prime} Q e_{321}+2\left(\operatorname{sgn}\left(l_{k}^{*}\right) b_{m}^{\prime} P e_{321} x_{1}^{\prime}+\gamma^{-1} \dot{\tilde{k}}_{31}^{\prime}\right) \frac{\tilde{k}_{31}^{\prime}}{\left|l_{3}^{*}\right|}+2\left(\operatorname{sgn}\left(l_{3}^{*}\right) b_{m}^{\prime} P e_{321} x_{2}^{\prime}+\gamma^{-1} \dot{\tilde{k}}_{32}^{\prime}\right) \frac{\tilde{k}_{32}^{\prime}}{\left|l_{3}^{*}\right|} \\
& +2\left(\operatorname{sgn}\left(l_{3}^{*}\right) b_{m}^{\prime} P e_{321} e_{321}^{\prime}+\gamma^{-1} \dot{\tilde{k}}_{3}^{\prime}\right) \frac{\tilde{k}_{3}}{\left|l_{3}^{*}\right|}+2\left(\operatorname{sgn}\left(l_{3}^{*}\right) b_{m}^{\prime} P e_{321} u_{1}(t)+\gamma^{-1} \dot{\tilde{l}}_{31}\right) \frac{\tilde{l}_{31}^{\prime}}{\left|l_{3}^{*}\right|} \\
& +2\left(\operatorname{sgn}\left(l_{3}^{*}\right) b_{m}^{\prime} P e_{321} u_{2}(t)+\gamma^{-1} \dot{\tilde{l}}_{32}\right) \frac{\tilde{l}_{32}^{\prime}}{\left|l_{3}^{*}\right|} \\
= & -e_{321}^{\prime} Q e_{321}
\end{aligned}
$$

where we have used (25). Using similar Lyapunov arguments as before one can prove $e_{321} \rightarrow 0$. This shows that the state of agent 3 converges to the average of the states of agents 1 and 2 . If the states of agents 1 and 2 converge to the state $x_{m}$ of a reference model (e.g. because they are connected via a directed tree to a root node that has access to the reference signal $r$ ), then the state of agent 3 will converge to the same reference state $x_{m}$.

\subsection{General case}

In the general case, we consider a set of $N$ agents

$$
\dot{x}_{i}=A_{i} x_{i}+b_{i} u_{i}, \quad i \in\{1, \ldots, N\}
$$

where agent 1 is the one that can access the reference $r$ in (2). Assumptions 1 and 2 should be revised as follows.

Assumption (rev.) 1. For every agent $i$, there exist a constant vector $k_{i}^{*}$ and scalar $l_{i}^{*}$ such that

$$
A_{m}=A_{i}+b_{i} k_{i}^{* \prime}, \quad b_{m}=b_{i} l_{i}^{*}
$$

Assumption (rev.) 2. For every agent $i$, the signs of $l_{i}^{*}$ is known. The sign can eventually be different for different agents. 
Remark 5. Assumptions rev. 1 and rev. 2 generalize Assumptions 1 and 2 to the multiagent setting. Since the agents are unknown and heterogeneous, Assumption rev. 1 is required to make the behavior of the agents homogeneous via appropriate gains. Therefore, (30) contains the 'feedback matching conditions' for all agents. With respect to Assumption rev. 2, it is worth mentioning that often, despite uncertainty, the sign of the input vector field is the same for all agents (e.g. in nature the sign of the input vector field defined by wings or fins is the same for the entire flock, or in technological flocks of robots/vehicles the input vector field defined by gas and braking inputs is the same for the entire flock). As a result, the sign of $l_{i}^{*}$ is often the same for all $i$. Finally, similarly to Proposition 1 , one can verify that Assumptions rev. 1 implies the existence, for every pair of agents $(i, j)$, of a constant vector $k_{j i}^{*}$ and a scalar $l_{j i}^{*}$ such that

$$
A_{i}=A_{j}+b_{j} k_{j i}^{* \prime}, \quad b_{j}=b_{2} l_{j i}^{*}
$$

which implies that every agent can be matched to its neighbor via appropriate gains. Therefore, (31) contains the 'coupling matching conditions' for all agents. In fact, we have shown in the previous examples that in order to achieve synchronization, heterogeneity has to be 'canceled' via feedback and coupling gains. We refer to $k_{i}^{*}$, $l_{i}^{*}$ as feedback gains, and to $k_{j i}^{*}, l_{j i}^{*}$ as coupling gains.

Together with Assumptions rev. 1 and rev. 2, we make the following assumption of the communication topology.

Assumption 3. The directed communication graph is acyclic. In addition, the graph contains a directed spanning tree with the leader as the root node.

Remark 6. The first part of Assumption 3 is equivalent to saying that the vertices of the graph can be sorted in such a way that the adjacency matrix has upper triangular form with only zeros in the diagonal.

Except for agent 1, which uses controller (6) and adaptive laws (7), the following controller is proposed for the other agents

$$
u_{j}(t)=\frac{\sum_{i=1}^{N} a_{i j} k_{j i}^{\prime}(t) x_{i}(t)}{\sum_{i=1}^{N} a_{i j}}+k_{j}^{\prime}(t) \frac{\sum_{i=1}^{N} a_{i j}\left(x_{j}(t)-x_{i}(t)\right)}{\sum_{i=1}^{N} a_{i j}}+\frac{\sum_{i=1}^{N} a_{i j} l_{j i}(t) u_{i}(t)}{\sum_{i=1}^{N} a_{i j}}
$$

with the adaptive laws

$$
\begin{aligned}
\dot{k}_{j i}^{\prime}(t) & =-\operatorname{sgn}\left(l_{j}^{*}\right) \gamma b_{m}^{\prime} P\left[\sum_{i=1}^{N} a_{i j}\left(x_{j}(t)-x_{i}(t)\right)\right] x_{i}^{\prime}(t) \\
\dot{k}_{j}^{\prime}(t) & =-\operatorname{sgn}\left(l_{j}^{*}\right) \gamma b_{m}^{\prime} P\left[\sum_{i=1}^{N} a_{i j}\left(x_{j}(t)-x_{i}(t)\right)\right]\left[\sum_{i=1}^{N} a_{i j}\left(x_{j}(t)-x_{i}(t)\right)\right]^{\prime} \\
\dot{l}_{j i}(t) & =-\operatorname{sgn}\left(l_{j}^{*}\right) \gamma b_{m}^{\prime} P\left[\sum_{i=1}^{N} a_{i j}\left(x_{j}(t)-x_{i}(t)\right)\right] u_{i}(t)
\end{aligned}
$$

where $k_{j i}, k_{i}, l_{j i}$ are the estimates of $k_{j i}^{*}, k_{i}^{*}, l_{j i}^{*}$ respectively.

One can verify that, with the appropriate adjacency matrices, the adaptive controller (32)-(33) reduces to the special cases (14)-(15) or (24)-(25). Like in the two-agent and three-agent cases, the resulting adaptive law (32)-(33) does not exploit any measurements of $r$, but it is based on measurements of $u_{j}$ to be used as a feedforward term.

The following result can be stated:

Theorem 1. Consider the unknown linear systems (29), with reference model (2), controllers (6), (32), and update laws (7), (33). Then, all closed-loop signals are bounded and $e_{i}=x_{i}-x_{m}, e_{j i}=x_{j}-x_{i}$, with $i, j$ such that $a_{i j} \neq 0$, converge asymptotically to zero.

PROOF. The proof uses similar tools as before with the Lyapunov function

$$
V=\sum_{j=1}^{N}\left[\sum_{i=0}^{N} a_{i j} e_{j i}\right]^{\prime} P\left[\sum_{i=0}^{N} a_{i j} e_{j i}\right]+\sum_{j=1}^{N} \operatorname{tr}\left[\frac{\tilde{k}_{j}^{\prime} \tilde{k}_{j}}{\gamma\left|l_{j}^{*}\right|}\right]+\sum_{j=1}^{N} \sum_{i=1}^{N} a_{i j} t r\left[\frac{\tilde{k}_{j i}^{\prime} \tilde{k}_{j i}}{\gamma\left|l_{j}^{*}\right|}\right]+\sum_{j=1}^{N} \sum_{i=1}^{N} a_{i j} \frac{\tilde{l}_{j i}^{2}}{\gamma\left|l_{j}^{*}\right|}
$$


where the index $i=0$ has been used as a short notation for the reference model, i.e. $e_{j 0}=e_{j}=x_{j}-x_{m}$ and $a_{j 0} \neq 0$ only for the root node. Furthermore, from (29) and (2), we can obtain the following dynamics of the error

$$
\begin{aligned}
\dot{e}_{j i} & =A_{m}\left(x_{j}-x_{i}\right)+b_{j}\left[u_{j}-k_{j i}^{* \prime} x_{i}-k_{j}^{* \prime} e_{j i}-l_{j i}^{*} u_{i}\right] \\
& =A_{m} e_{j i}+b_{j}\left[\tilde{k}_{j i}^{\prime} x_{i}+\tilde{k}_{j}^{\prime} e_{j i}+\tilde{l}_{j i} u_{i}\right] .
\end{aligned}
$$

The attentive reader will have recognized a similar structure as in the previously developed two-agent and three-agent cases. The time derivative of (34) along (35) can be worked out accordingly

$$
\begin{aligned}
\dot{V}= & \sum_{j=1}^{N}\left[\sum_{i=0}^{N} a_{i j} e_{j i}\right]^{\prime}\left(P A_{m}+A_{m}^{\prime} P\right)\left[\sum_{i=0}^{N} a_{i j} e_{j i}\right]+2\left[\sum_{i=0}^{N} a_{i j} e_{j i}\right]^{\prime} P b_{j}\left[\sum_{j=1}^{N} a_{i j} \tilde{k}_{j i}^{\prime} x_{i}+\tilde{k}_{j}^{\prime} \sum_{j=1}^{N} a_{i j} e_{j i}+\sum_{j=1}^{N} a_{i j} \tilde{l}_{j i} u_{i}\right] \\
& +\sum_{j=1}^{N} \operatorname{tr}\left[\frac{\tilde{k}_{j}^{\prime} \gamma^{-1} \dot{\tilde{k}}_{j}}{\left|l_{j}^{*}\right|}\right]+\sum_{j=1}^{N} \sum_{i=1}^{N} a_{i j} t r\left[\frac{\left.\tilde{k}_{j i}^{\prime} \gamma^{-1} \dot{\tilde{k}}_{j i}\right]}{\left|l_{j}^{*}\right|}\right]+\sum_{j=1}^{N} \sum_{i=1}^{N} a_{i j} \frac{\tilde{l}_{j i} \gamma^{-1} \dot{\tilde{l}}_{j i}}{\left|l_{j}^{*}\right|} \\
= & -\sum_{j=1}^{N}\left[\sum_{i=0}^{N} a_{i j} e_{j i}\right]^{\prime} Q\left[\sum_{i=0}^{N} a_{i j} e_{j i}\right]
\end{aligned}
$$

where we have used (33). Convergence of the errors $e_{j i}$ to zero can be proved using Barbalat's Lemma, while convergence of $x_{i}-x_{m}$ to zero follows accordingly using Assumption 3 .

Remark 7. In literature with adaptive couplings (see e.g. [8, 23]), the scalar coupling weight is updated via the scalar quantity $e_{j i}^{\prime} e_{j i}$. On the other hand, the controller (32) reveals the structure of the synchronization task: each agent needs vector-valued update laws (e.g. note the presence of $e_{j i} e_{j i}^{\prime}$ in the update of $k_{i}$ in (33) to estimate the feedback gains $k_{i}$ to match agent $i$ to the reference model, and the coupling weights $k_{j i}, l_{j i}$ to match agent $j$ the neighbors $i$ : thus the synchronization law (32)-(33) implicitly includes the update of both feedback and the coupling gains arising from distributed matching conditions, while most synchronization laws in literature consider a fixed feedback gain based on some nominal model of the agent and updates the coupling weight only (e.g. [25, 29]).

\section{Robust adaptive leader-follower synchronization}

In the previous section we have assumed that each agent is able to evaluate exactly the input of each one of its neighbors. In practice, it is reasonable to assume that some bounded error will occur in the evaluation of such input. This error will be referred to as misjudgment error, which leads to the agent model

$$
\dot{x}_{j}=A_{j} x_{i}+b_{j}\left[\frac{\sum_{i=1}^{N} a_{i j} k_{j i}^{\prime}(t) x_{i}(t)}{\sum_{i=1}^{N} a_{i j}}+k_{j}^{\prime}(t) \frac{\sum_{i=1}^{N} a_{i j}\left(x_{j}(t)-x_{i}(t)\right)}{\sum_{i=1}^{N} a_{i j}}+\frac{\sum_{i=1}^{N} a_{i j} l_{j i}(t) u_{i}(t)}{\sum_{i=1}^{N} a_{i j}}+\frac{\sum_{i=1}^{N} a_{i j} d_{j i}}{\sum_{i=1}^{N} a_{i j}}\right]
$$

where $d_{j i}$ is the misjudgment error which is assumed to be bounded, i.e. $\left|d_{j i}\right| \leq \bar{d}$, for some unknown $\bar{d}$. In order to handle the disturbance term, the following leakage-based adaptation law is proposed

$$
\begin{aligned}
\dot{k}_{j i}^{\prime}(t) & =-\operatorname{sgn}\left(l_{j}^{*}\right) \gamma b_{m}^{\prime} P\left[\sum_{i=1}^{N} a_{i j}\left(x_{j}(t)-x_{i}(t)\right)\right] x_{i}^{\prime}(t)-\operatorname{sgn}\left(l_{j}^{*}\right) \sigma \gamma k_{j i}^{\prime} \\
\dot{k}_{j}^{\prime}(t) & =-\operatorname{sgn}\left(l_{j}^{*}\right) \gamma b_{m}^{\prime} P\left[\sum_{i=1}^{N} a_{i j}\left(x_{j}(t)-x_{i}(t)\right)\right]\left[\sum_{i=1}^{N} a_{i j}\left(x_{j}(t)-x_{i}(t)\right)\right]^{\prime}-\operatorname{sgn}\left(l_{j}^{*}\right) \sigma \gamma k_{i}^{\prime} \\
\dot{l}_{j i}(t) & =-\operatorname{sgn}\left(l_{j}^{*}\right) \gamma b_{m}^{\prime} P\left[\sum_{i=1}^{N} a_{i j}\left(x_{j}(t)-x_{i}(t)\right)\right] u_{i}(t)-\operatorname{sgn}\left(l_{j}^{*}\right) \sigma \gamma l_{j i}
\end{aligned}
$$

where $\sigma>0$ is a scalar that allows robustness with respect to disturbances. In fact, the following result can be stated 
Theorem 2. Consider a flock composed of unknown heterogeneous linear agents (37), with reference model (2), controller (32), and adaptive laws (38). Then all closed-loop signals are bounded and all $e_{j i}$ are of the order of $\bar{d}$.

Proof. Consider the same candidate Lyapunov function (34). From (37) and (2), we obtain the following dynamics of the error

$$
\dot{e}_{j i}=A_{m} e_{j i}+b_{j}\left[\tilde{k}_{j i}^{\prime} x_{i}+\tilde{k}_{j}^{\prime} e_{j i}+\tilde{l}_{j i} u_{i}+d_{j i}\right]
$$

where we have used the matching condition (30). The time derivative of the Lyapunov function (34) along the trajectory of the error (39) turns out to be

$$
\begin{aligned}
\dot{V}= & \sum_{j=1}^{N}\left[\sum_{i=0}^{N} a_{i j} e_{j i}\right]^{\prime}\left(P A_{m}+A_{m}^{\prime} P\right)\left[\sum_{i=0}^{N} a_{i j} e_{j i}\right]+2\left[\sum_{i=0}^{N} a_{i j} e_{j i}\right]^{\prime} P b_{j}\left[\sum_{j=1}^{N} a_{i j} \tilde{k}_{j i}^{\prime} x_{i}+\tilde{k}_{j}^{\prime} \sum_{j=1}^{N} a_{i j} e_{j i}+\sum_{j=1}^{N} a_{i j} \tilde{l}_{j i} u_{i}\right] \\
& +\sum_{j=1}^{N} \operatorname{tr}\left[\frac{\tilde{k}_{j}^{\prime} \gamma^{-1} \dot{\tilde{k}}_{j}}{\left|l_{j}^{*}\right|}\right]+\sum_{j=1}^{N} \sum_{i=1}^{N} a_{i j} t r\left[\frac{\tilde{k}_{j i}^{\prime} \gamma^{-1} \dot{\tilde{k}}_{j i}}{\left|l_{j}^{*}\right|}\right]+\sum_{j=1}^{N} \sum_{i=1}^{N} a_{i j} \frac{\tilde{l}_{j i} \gamma^{-1} \dot{\tilde{l}}_{j i}}{\left|l_{j}^{*}\right|} \\
=- & -\sum_{j=1}^{N}\left[\sum_{i=0}^{N} a_{i j} e_{j i}\right]^{\prime} Q\left[\sum_{i=0}^{N} a_{i j} e_{j i}\right] \\
& +\sum_{j=1}^{N}\left[-2 \frac{\sigma}{\left|l_{j}^{*}\right|} \tilde{k}_{j} k_{j}^{\prime}+\sum_{i=0}^{N} a_{i j}\left(-2 \frac{\sigma}{\left|l_{j}^{*}\right|} \tilde{l}_{j i} l_{j i}^{\prime}-2 \frac{\sigma}{\left|l_{j}^{*}\right|} \tilde{k}_{j i} k_{j i}^{\prime}+2 \frac{\sigma}{\left|l_{j}^{*}\right|} b_{m}^{\prime} P e_{j i} d_{j i}\right)\right]
\end{aligned}
$$

where we have used (38). Using the inequality $-a^{\prime} a+a^{\prime} b \leq-a^{\prime} a / 2+b^{\prime} b / 2$ for any $a, b$, we write

$$
\begin{aligned}
\dot{V} \leq-\sum_{j=1}^{N} & {\left[\sum_{i=0}^{N} a_{i j} e_{j i}\right]^{\prime} Q\left[\sum_{i=0}^{N} a_{i j} e_{j i}\right]+\sum_{j=1}^{N}-\frac{\sigma}{\left|l_{j}^{*}\right|} k_{j} k_{j}^{\prime}+\frac{\sigma}{\left|l_{j}^{*}\right|} k_{j}^{*} k_{j}^{* \prime} } \\
+ & {\left[\sum_{i=0}^{N} a_{i j}\left(-\frac{\sigma}{\left|l_{j}^{*}\right|} k_{j i} k_{j i}^{\prime}+\frac{\sigma}{\left|l_{j}^{*}\right|} k_{j i}^{*} k_{j i}^{* \prime}-\frac{\sigma}{\left|l_{j}^{*}\right|} l_{j i} l_{j i}^{\prime}+\frac{\sigma}{\left|l_{j}^{*}\right|} l_{j i}^{*} l_{j i}^{* \prime}+2 \frac{\sigma}{\left|l_{j}^{*}\right|} b_{m}^{\prime} P e_{j i} d_{j i}\right)\right] }
\end{aligned}
$$

Using standard Lyapunov reasoning [38, Chap. 4], we observe that, if $k_{j i} k_{j i}^{\prime}+k_{j} k_{j}^{\prime}+l_{j i}^{2} \geq k_{j i}^{*} k_{j i}^{* 1}+k_{j}^{*} k_{j}^{* \prime}+l_{j i}^{* 2}+$ $2 b_{m}^{\prime} P e_{j i} \bar{d}, \dot{V}$ will be negative definite (and decreasing exponentially), from which we conclude that $e_{j i}$ will converge inside a ball with radius proportional to $\bar{d}$.

\subsection{Leader-follower synchronization with input estimation}

Finally, an interesting situation to be studied is what would happen if no input $u_{i}$ would be available to agent $j$. We will discuss the simplest case of constant but unknown leader input (this implies a constant reference $r$ ). In this case we propose to estimate the input $u_{j}$ from each agent, which leads to the control

$$
u_{j}(t)=\frac{\sum_{i=1}^{N} a_{i j} k_{j i}^{\prime}(t) x_{i}(t)}{\sum_{i=1}^{N} a_{i j}}+k_{j}^{\prime}(t) \frac{\sum_{i=1}^{N} a_{i j}\left(x_{j}(t)-x_{i}(t)\right)}{\sum_{i=1}^{N} a_{i j}}+\frac{\sum_{i=1}^{N} a_{i j} \hat{u}_{i j}(t)}{\sum_{i=1}^{N} a_{i j}}
$$

and to the agent model

$$
\dot{x}_{j}=A_{j} x_{i}+b_{j}\left[\frac{\sum_{i=1}^{N} a_{i j} k_{j i}^{\prime} x_{i}}{\sum_{i=1}^{N} a_{i j}}+k_{j}^{\prime} \frac{\sum_{i=1}^{N} a_{i j}\left(x_{j}-x_{i}\right)}{\sum_{i=1}^{N} a_{i j}}+\frac{\sum_{i=1}^{N} a_{i j} \hat{u}_{i j}}{\sum_{i=1}^{N} a_{i j}}\right]
$$

where $\hat{u}_{i_{j}}$ represents the estimate of $u_{i}$ calculated by agent $j$, and the term $l_{j i}$ has been removed since its estimate is included in the estimate $\hat{u}_{i_{j}}$. While $k_{j i}$ and $k_{j}$ are updated as in (32), we propose an extra estimator for $\hat{u}_{i j}$

$$
\dot{\hat{u}}_{i_{j}}=-\operatorname{sgn}\left(l_{j}^{*}\right) \gamma b_{m}^{\prime} P\left[\sum_{i=1}^{N} a_{i j}\left(x_{j}(t)-x_{i}(t)\right)\right]
$$


It has to be underlined that each agent $j$ will have its own estimate $\hat{u}_{i_{j}}$ for the estimate of its neighbor, so the estimation is completely distributed and does not need extra communication.

Theorem 3. Consider a flock composed of unknown heterogeneous linear agents (43), with reference model (2) with constant reference signal, controller (42) and update laws (32) and (44). Then all closed-loop signals are bounded and $e_{i}=x_{i}-x_{m}, e_{j i}=x_{j}-x_{i}$, with $i, j$ such that $a_{i j} \neq 0$, converge asymptotically to zero.

Proof. Consider the candidate Lyapunov function

$$
V=\sum_{j=1}^{N}\left[\sum_{i=0}^{N} a_{i j} e_{j i}\right]^{\prime} P\left[\sum_{i=0}^{N} a_{i j} e_{j i}\right]+\sum_{j=1}^{N} \operatorname{tr}\left[\frac{\tilde{k}_{j}^{\prime} \tilde{k}_{j}}{\gamma\left|l_{j}^{*}\right|}\right]+\sum_{j=1}^{N} \sum_{i=1}^{N} a_{i j} \operatorname{tr}\left[\frac{\tilde{k}_{j i}^{\prime} \tilde{k}_{j i}}{\gamma\left|l_{j}^{*}\right|}\right]+\sum_{j=1}^{N} \sum_{i=1}^{N} a_{i j} \frac{\tilde{u}_{i j}^{2}}{\gamma\left|l_{j}^{*}\right|}
$$

where $\tilde{u}_{i j}=\hat{u}_{i_{j}}-u_{i}$. For the sake of clarification, it is important to mention that $u_{i}$ represents the actual input of agent $i$ and $\hat{u}_{i j}$ represents its estimate calculated by agent $j$. Furthermore, from (30), we can obtain the following dynamics of the error

$$
\dot{e}_{j i}=A_{m} e_{j i}+b_{j}\left[\tilde{k}_{j i}^{\prime} x_{i}+\tilde{k}_{j}^{\prime} e_{j i}+\tilde{u}_{i j}\right]
$$

The time derivative of the Lyapunov function (45) along the trajectories (46) turns out to be

$$
\begin{aligned}
\dot{V}= & \sum_{j=1}^{N}\left[\sum_{i=0}^{N} a_{i j} e_{j i}\right]^{\prime}\left(P A_{m}+A_{m}^{\prime} P\right)\left[\sum_{i=0}^{N} a_{i j} e_{j i}\right]+2\left[\sum_{i=0}^{N} a_{i j} e_{j i}\right]^{\prime} P b_{j}\left[\sum_{j=1}^{N} a_{i j} \tilde{k}_{j i}^{\prime} x_{i}+\tilde{k}_{j}^{\prime} \sum_{j=1}^{N} a_{i j} e_{j i}+\sum_{j=1}^{N} a_{i j} \tilde{u}_{i j}\right] \\
& +\sum_{j=1}^{N} \operatorname{tr}\left[\frac{\tilde{k}_{j}^{\prime} \gamma^{-1} \dot{\tilde{k}}_{j}}{\left|l_{j}^{*}\right|}\right]+\sum_{j=1}^{N} \sum_{i=1}^{N} a_{i j} t r\left[\frac{\tilde{k}_{j i}^{\prime} \gamma^{-1} \dot{\tilde{k}}_{j i}}{\left|l_{j}^{*}\right|}\right]+\sum_{j=1}^{N} \sum_{i=1}^{N} a_{i j}\left[\frac{\tilde{u}_{i j} \gamma^{-1} \dot{\tilde{u}}_{i j}}{\left|l_{i}^{*}\right|}\right] \\
= & -\sum_{j=1}^{N}\left[\sum_{i=0}^{N} a_{i j} e_{j i}\right]^{\prime} Q\left[\sum_{i=0}^{N} a_{i j} e_{j i}\right]
\end{aligned}
$$

where we have used (32) and (44). We conclude convergence of the synchronization error to zero using Barbalat's Lemma (to prove converge of $e_{j i}$ ) and Assumption 3 (to prove convergence of $x_{i}-x_{m}$ ).

\section{Motivational example: cooperative adaptive cruise control}

Let us introduce platooning as a possible application field of the proposed algorithms. Platooning is an automated driving method in which vehicles are grouped into platoons, where the speed of each vehicle (except eventually the speed of the leading vehicle) is automatically adjusted so as to maintain a safe inter-vehicle distance. The most celebrated platooning technology is Cooperative Adaptive Cruise Control (CACC), an extension of Adaptive Cruise Control (ACC) where platooning is enabled by inter-vehicle communication in addition to on-board sensors [33]. A simple description of the CACC setting (for longitudinal dynamics only) is given as follows: by taking as a state the vehicle velocity and its position minus the desired spacing, vehicle $i$ can be modelled by the following linear system

$$
\dot{x}_{i}=\left[\begin{array}{cc}
0 & 1 \\
a_{1_{i}} & a_{2_{i}}
\end{array}\right] x_{i}+\left[\begin{array}{c}
0 \\
b_{1_{i}}
\end{array}\right] u_{i}
$$

where $a_{1_{i}}$ and $a_{2_{i}}$ contain some driveline parameters, and $b_{1_{i}}$ contains the efficiency of the engine. All parameters are often uncertain and influenced by road conditions (e.g. weather, contact road-wheel, road slope). The input $u_{i}$ has the dimension of an acceleration (or of a force, when multiplied by the vehicle mass ). As the driveline and engine parameters are different for every vehicle, we have to deal with heterogeneous agents. The leading vehicle (indexed with 1) is in charge of following a certain acceleration profile (which can be the result of a reference model indexed with 0 ), while the other vehicles (indexed with $2, \ldots, N$ ) should keep a desired spacing and reduce the relative velocity to zero, a task which can be described in terms of synchronization as $x_{j}-x_{i} \rightarrow 0$. If vehicles were equipped with only on-board sensors, we would have the so-called ACC setting in which the relative position and relative velocity (i.e. 
$x_{j}-x_{i}$ ) can be measured (via laser sensor), and consequently, by knowing its own position and velocity, vehicle $i$ can derive the state of a preceding $j$. Note that in this case one would have a one-step look-ahead topology in which a vehicle can interact only with the preceding vehicle.

In CACC one adds inter-vehicle communication to the ACC: in other words, a vehicle can communicate via wireless sensors its own acceleration to the following vehicle (or vehicles), so that this information can be used to improve platooning performance. In fact, while string stability ${ }^{2}$ in ACC strategies cannot be guaranteed for intervehicle time gaps smaller than 1 second, CACC was shown to guarantee string stability for time gaps significantly smaller than 1 second $[39,40]$. In CACC settings it might make sense to overcome the one-step look-ahead topology with multi-step look-ahead topology, in which a following vehicle can receive information from two or more preceding vehicles. In practice, however, the one-step look-ahead topology is the most popular topology even in CACC, because looking too many vehicles ahead increases communication delays and decreases communication reliability [41].

It is now straightforward to realize that the proposed adaptive synchronization setting has a direct technological application in CACC. Let us focus on the one-step look-ahead topology: in CACC the control law of vehicle $i$ takes usually the form

$$
u_{i}(t)=k_{i, i-1}^{\prime}(t) x_{i-1}(t)+k_{i}^{\prime}(t)\left(x_{i}(t)-x_{i-1}(t)\right)+l_{i, i-1}(t) u_{i-1}(t)
$$

which, similarly to (32), involves measurements of relative position and relative velocity $x_{i}(t)-x_{i-1}(t)$, position and velocity of the preceding vehicle $x_{i-1}$ and acceleration of the preceding vehicle $u_{i-1}$. Therefore, the proposed adaptive laws have a relevant motivation: only recently research has been addressing the problem of platooning with heterogeneous vehicles [42, 43], while previously the homogeneous case (identical vehicle dynamics) was the main focus: intuitively, the heterogeneity of the driveline dynamics, combined with the fact that vehicle parameters might be unknown or even change with time motivates an adaptive control approach. The following section will illustrate some experiments involving second-order agents as the ones in (48): we will adopt an acyclic topology more complex then the one-step look ahead topology, in order to show more generality than the platooning case.

\section{Simulations}

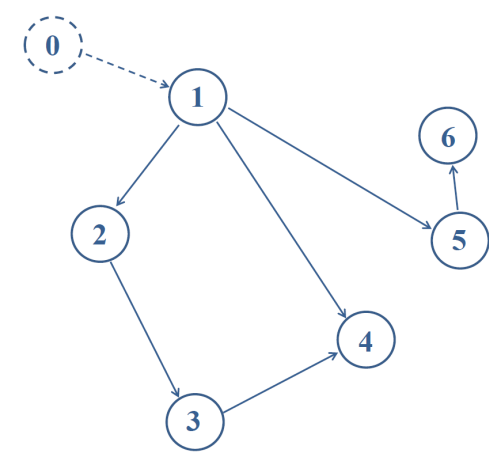

Figure 1: The leader-follower directed communication graph

The simulations are carried out on the directed graph shown in Fig. 1, where node 1 acts as the leader node. The reference model is indicated as a fictitious agent 0 which can communicate the reference signal to agent 1 . The agents are taken as second-order linear agent in the canonical form, where the leader dynamics and the dynamics of other agents take the form (48), where the exact coefficients and initial conditions are reported in Table 1. Note that the matrices are given in terms of $\dot{x}_{0}=A_{m} x_{0}+b_{m} r$ for the reference model and $\dot{x}_{i}=A_{i} x_{i}+b_{i} u_{i}, i \in\{1, \ldots, N\}$ for the other agents. Except for the reference model, which is asymptotically stable, all agents are open-loop unstable, so that the couplings must also take care of stabilizing the agents. In addition, all agents are heterogeneous, and the numerical

\footnotetext{
${ }^{2}$ String stability is a desired behavior of the platoon, fulfilled when the effect of disturbances (e.g. emergency braking) introduced along the platoon is attenuated as it propagates in the upstream direction.
} 
values in Table 1 are used only to simulate the agents, but the synchronization protocol uses only the knowledge of $A_{m}$ and $b_{m}$, and not of the other matrices. The other design parameters are taken as: $\gamma=10, Q=\operatorname{diag}(100,1)$, and all coupling vector gains are initialized to be 0 . Also note that $\operatorname{sgn}\left(l_{i}^{*}\right)=1, \forall i$.

\begin{tabular}{c|c|c|c|c} 
& $a_{1}$ & $a_{2}$ & $b_{1}$ & $x_{0}$ \\
\hline \hline agent \#0 & -0.5 & -1 & 1 & {$\left[\begin{array}{ll}1 & -1\end{array}\right]^{\prime}$} \\
agent \#1 & -1 & 2 & 1 & {$\left[\begin{array}{ll}1 & 1\end{array}\right]^{\prime}$} \\
agent \#2 & -0.75 & 2.5 & 0.5 & {$\left[\begin{array}{ll}-1 & -1\end{array}\right]^{\prime}$} \\
agent \#3 & -1.25 & 2 & 1.25 & {$\left[\begin{array}{ll}-1 & 0\end{array}\right]^{\prime}$} \\
agent \#4 & -0.5 & 1 & 0.75 & {$\left[\begin{array}{ll}0 & 1\end{array}\right]^{\prime}$} \\
agent \#5 & -0.75 & 1 & 1.5 & {$\left[\begin{array}{ll}1 & 0\end{array}\right]^{\prime}$} \\
agent \#6 & -1.5 & 2.5 & 1 & {$\left[\begin{array}{ll}-1 & 1\end{array}\right]^{\prime}$}
\end{tabular}

Table 1: Coefficients and initial conditions of the agents

(a) Synchronization in noiseless case. In these simulations we assume that each agent can observe perfectly the input of its neighbor. The resulting synchronization is shown in Fig. 2a and Fig. 2b for a sinusoidal and a constant leader input respectively. It is observed that all states converge asymptotically to the state of the leader.
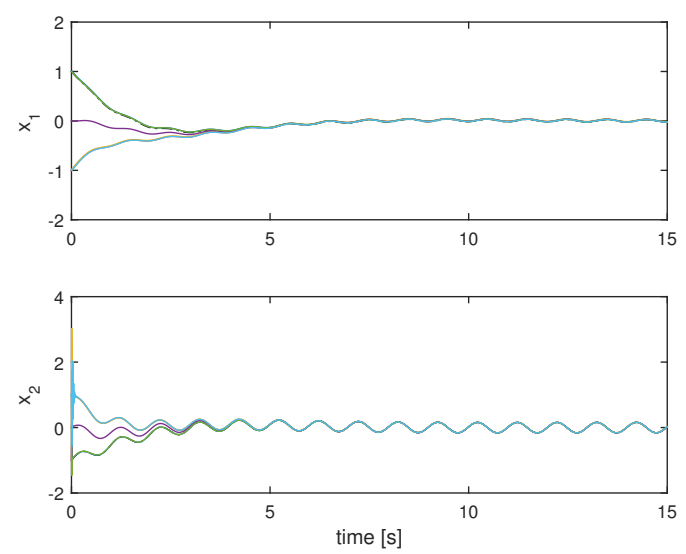

(a) sinusoidal input
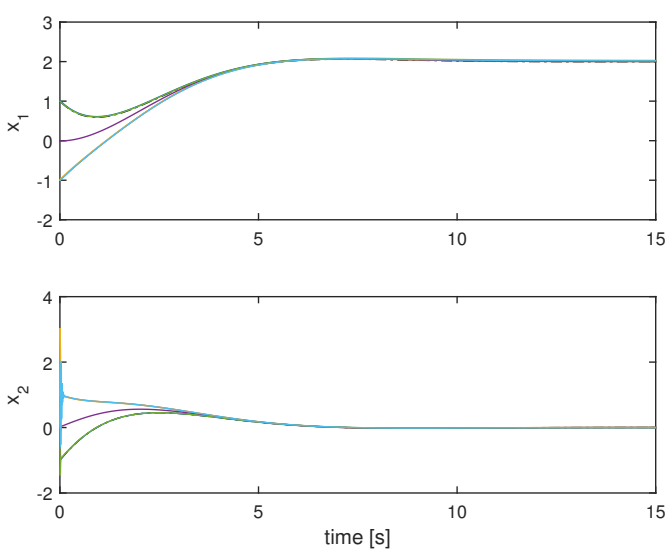

(b) constant input

Figure 2: Synchronization in noiseless case

(b) Fixed-gain feedback. In these simulations we use the following nominal agent $a_{1}=-0.95, a_{2}=1.83, b_{1}=1$ (calculated from the average of all agents) in order to design a fixed-gain feedback. The resulting state is shown in Fig. $3 a$ and Fig. $3 b$ for a sinusoidal and a constant leader input respectively. It is observed that, in the absence of vectorvalued adaptation, the fixed gain does not cancel the heterogeneities perfectly: this means that not all agents cannot match the reference model, and asymptotic convergence is in general not achieved. These simulations underline the need for adaptive feedback gains.

(c) Synchronization with bounded misjudgment error. In these simulations we assume that each agent can observe the input of its neighbor, but with a certain bounded misjudgment error. The misjudgment error is taken to be a random value with uniform distribution in $[-1,1]$. For these simulations the leakage parameter is taken as $\sigma=0.1$. The resulting synchronization is shown in Fig. 4a and Fig. 4b for a sinusoidal and a constant leader input respectively. It is observed that all states converge to the state of the leader with a certain bounded error.

(d) Synchronization with unknown constant input. In this final simulation we assume that the input of the leader is constant, and each agent cannot observe the input of its neighbor (which also converges to a constant), but it must estimate it. So, for this simulation extra estimators for each agent must be implemented. The resulting synchronization is shown in Fig. 5a. It is observed that, despite the extra uncertainty, all states converge asymptotically to the state 

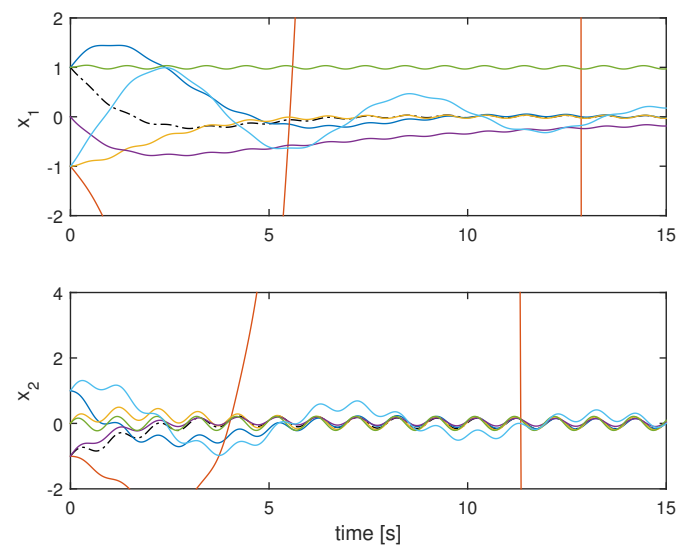

(a) sinusoidal input
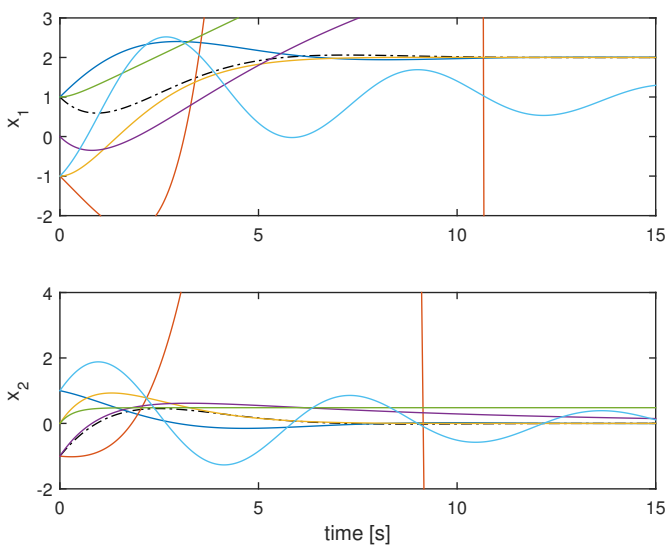

(b) constant input

Figure 3: Synchronization with fixed feedback gain
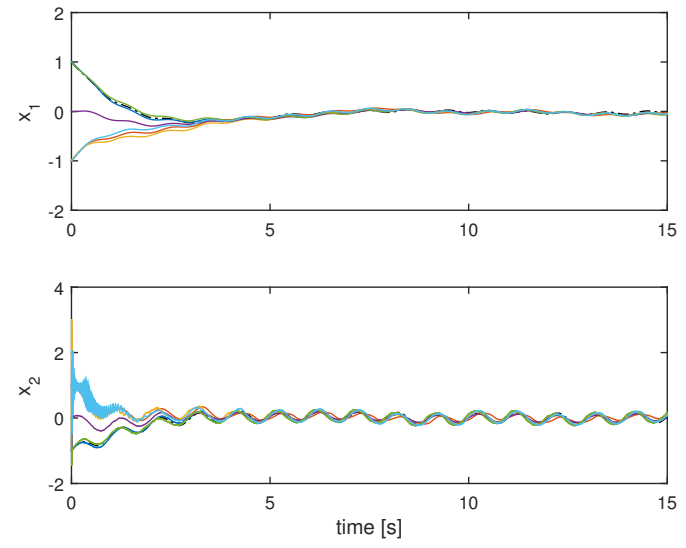

(a) sinusoidal input
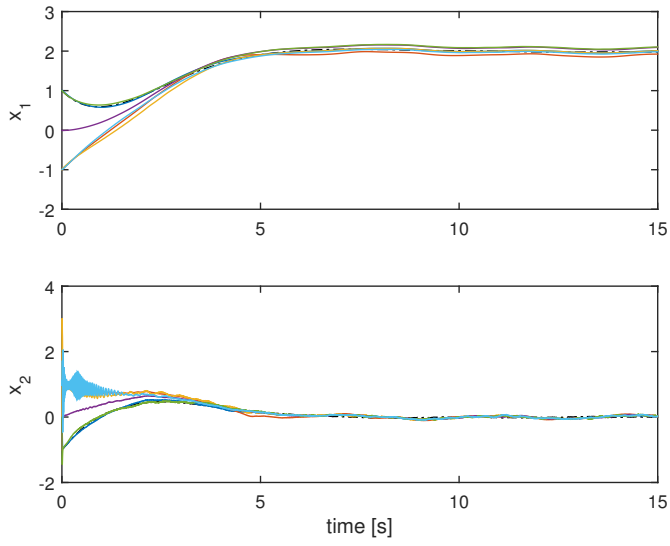

(b) constant input

Figure 4: Synchronization in noisy case

of the leader: this is achieved thanks to the extra estimators, whose state is shown in Fig. 5b. The estimation of $u_{1}$ is carried out from agent 2 and 5 (which are both connected to this agent): as typical in adaptive control, the estimate needs not to converge to the actual input (shown with a dashed line) for the synchronization error to go to zero.

\section{Conclusions}

This work presented an adaptive algorithm for leader-follower synchronization of heterogeneous and uncertain linear agents. The problem was solved by formulating the synchronization problem as a special model reference adaptive control where each agent tries to converge to the model defined by its neighbors. It has been shown that in this setting the 'feedback matching conditions', classical in adaptive control and defining a set of feedback gains for the agents, leads to 'coupling matching conditions' that define a set of coupling gains for the each agent to follow its neighbors. In the presence of uncertainty, both the feedback and the coupling gains have to be estimated. To this purpose, the useful concept of virtual reference has been introduced, which represents the fictitious reference signal that is followed by a neighboring agent. The implementation of a model reference adaptive control in this setting has lead to a set of adaptive laws for all gains: in contrast with most synchronization (or consensus) literature, where 

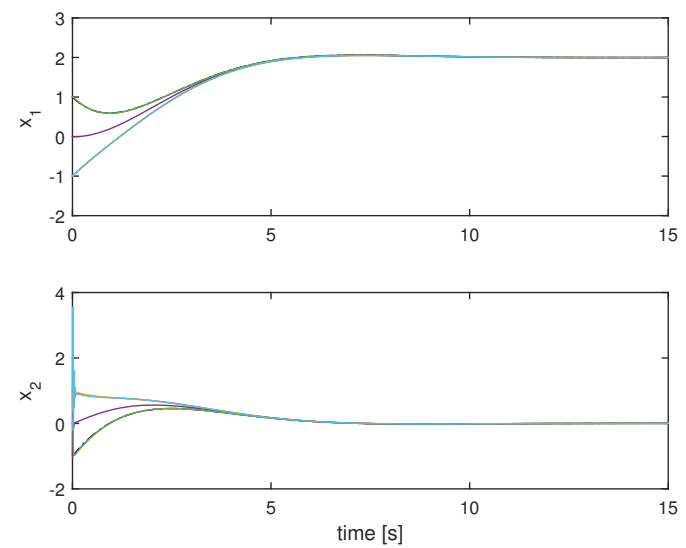

(a) States
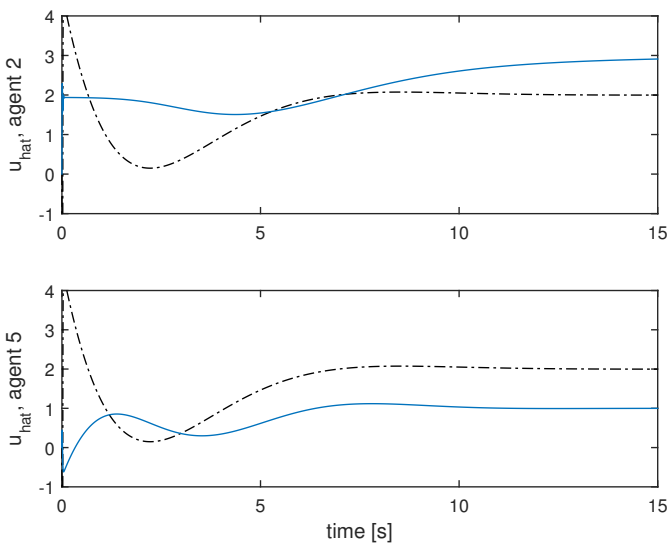

(b) estimation of $u_{1}$ from agent 2 and 5

Figure 5: Synchronization with unknown constant input

the adaptive laws are scalar-valued, in the proposed approach the adaptive laws are vector-valued. Therefore, in the presence of uncertainty, it was possible to overcome the fixed-gain control based on a nominal model of the agents. A Lyapunov-based approach was derived to show analytically that the synchronization error converges asymptotically to zero in the ideal noiseless case. A key feature of the algorithm was the knowledge of the input of the neighbors: this is assumed in many technological applications, most notably, in platooning via Cooperative Adaptive Cruise Control (CACC), which has been discussed as an engineering application of this work. If the input of the neighbors is measured with some (misjudgment) error or the input of the leader is unknown but constant, robust extensions of the approach have been provided, which rely on an adaptive law with leakage. The Lyapunov-based analysis reveals that the synchronization error will be bounded in the noisy case. Simulations have shown that the matching conditions allow all agents to converge to the same behavior: in the absence of adaptation, not all agents synchronize to the leader.

Future work will include extension to output-based synchronization and possibly consideration of unmatched uncertainties: in the presence of unmatched uncertainties we expect some bound on the synchronization error depending on the size of the unmatched uncertainty, in the spirit of [44]. Another promising research direction would be the possibility to handle switching topologies by using adaptive switching strategies similarly to [45, 46]. This research leaves also some open questions, the two most relevant being in our opinion the following. First, there might be a connection between communicating the input to neighbors (to construct the virtual reference as in the proposed approach) and communicating an auxiliary variable to neighbors (to observe the reference as in cooperative regulation schemes [24]): in both cases one has to communicate extra information in order to reconstruct the reference. Second, and related one, while communicating the input to neighbors seems to be sensitive to redundant information (cycles have to be carefully addressed [47]), the distributed observer scheme can handle cyclic information: it would be interesting to study new algorithms for communicating the input to neighbors, while being able to handle cycles.

\section{References}

[1] W. Ren, R. W. Beard, and E. M. Atkins, "A survey of consensus problems in multi-agent coordination," in Proceedings of the 2005, American Control Conference, 2005., 2005, pp. 1859-1864 vol. 3.

[2] - "Information consensus in multivehicle cooperative control," IEEE Control Systems, vol. 27, no. 2, pp. 71-82, 2007.

[3] R. Olfati-Saber, J. A. Fax, and R. M. Murray, "Consensus and cooperation in networked multi-agent systems," Proceedings of the IEEE, vol. 95, no. 1, pp. 215-233, 2007.

[4] F. Bullo, R. Carli, and P. Frasca, "Gossip coverage control for robotic networks: Dynamical systems on the space of partitions," SIAM Journal on Control and Optimization, vol. 50, no. 1, pp. 419-447, 2012.

[5] Z. Li, Z. Duan, G. Chen, and L. Huang, "Consensus of multiagent systems and synchronization of complex networks: A unified viewpoint," IEEE Transactions on Circuits and Systems I: Regular Papers, vol. 57, no. 1, pp. 213-224, 2010. 
[6] Y. A. Harfouch, S. Yuan, and S. Baldi, "An adaptive switched control approach to heterogeneous platooning with inter-vehicle communication losses," IEEE Transactions on Control of Network Systems, vol. PP, no. 99, pp. 1-1, 2017.

[7] F. Dorfler and F. Bullo, "Synchronization in complex networks of phase oscillators: A survey," Automatica, vol. 50, no. 6, pp. 1539 - 1564, 2014.

[8] L. F. R. Turci, P. De Lellis, E. E. N. Macau, M. Di Bernardo, and M. M. R. Simões, "Adaptive pinning control: A review of the fully decentralized strategy and its extensions," The European Physical Journal Special Topics, vol. 223, no. 13, pp. 2649-2664, 2014.

[9] R. Olfati-Saber and R. M. Murray, "Consensus problems in networks of agents with switching topology and time-delays," IEEE Transactions on Automatic Control, vol. 49, no. 9, pp. 1520-1533, 2004.

[10] W. Ren and R. W. Beard, "Consensus seeking in multiagent systems under dynamically changing interaction topologies," IEEE Transactions on Automatic Control, vol. 50, no. 5, pp. 655-661, 2005.

[11] W. Ren and E. Atkins, "Distributed multi-vehicle coordinated control via local information exchange," International Journal of Robust and Nonlinear Control, vol. 17, no. 10-11, pp. 1002-1033, 2007.

[12] W. Ren, "Consensus strategies for cooperative control of vehicle formations," IET Control Theory \& Applications, vol. 1, pp. $505-512,2007$.

[13] T. E. Gibson, "Adaptation and synchronization over a network: Asymptotic error convergence and pinning," in 2016 IEEE 55th Conference on Decision and Control (CDC), 2016, pp. 2969-2974.

[14] _ _ "Adaptation and synchronization over a network: Stabilization without a reference model," in 2016 IEEE 55th Conference on Decision and Control (CDC), 2016, pp. 2988-2993.

[15] C. W. Reynolds, "Flocks, herds and schools: A distributed behavioral model," SIGGRAPH Comput. Graph., vol. 21, no. 4, pp. $25-34,1987$.

[16] H. Hildenbrandt, C. Carere, and C. Hemelrijk, "Self-organized aerial displays of thousands of starlings: a model," Behavioral Ecology, vol. 21, no. 6, pp. 1349-1359, 2010.

[17] W. Ren, "Multi-vehicle consensus with a time-varying reference state," Systems \& Control Letters, vol. 56, no. 78, pp. 474 - 483, 2007.

[18] L. Cheng, Y. Wang, W. Ren, Z. G. Hou, and M. Tan, "On convergence rate of leader-following consensus of linear multi-agent systems with communication noises," IEEE Transactions on Automatic Control, vol. 61, no. 11, pp. 3586-3592, 2016.

[19] Z. Li, W. Ren, X. Liu, and L. Xie, "Distributed consensus of linear multi-agent systems with adaptive dynamic protocols," Automatica, vol. 49, no. 7, pp. $1986-1995,2013$.

[20] Z. Li, W. Ren, X. Liu, and M. Fu, "Consensus of multi-agent systems with general linear and lipschitz nonlinear dynamics using distributed adaptive protocols," IEEE Transactions on Automatic Control, vol. 58, no. 7, pp. 1786-1791, 2013.

[21] Z. Li and Z. Ding, "Distributed adaptive consensus and output tracking of unknown linear systems on directed graphs," Automatica, vol. 55, pp. $12-18,2015$

[22] Z. Ding and Z. Li, "Distributed adaptive consensus control of nonlinear output-feedback systems on directed graphs," Automatica, vol. 72, pp. $46-52,2016$.

[23] Z. Li, M. Z. Chen, and Z. Ding, "Distributed adaptive controllers for cooperative output regulation of heterogeneous agents over directed graphs," Automatica, vol. 68, pp. 179-183, 2016.

[24] G. S. Seyboth, W. Ren, and F. Allgower, "Cooperative control of linear multi-agent systems via distributed output regulation and transient synchronization," Automatica, vol. 68, pp. 132 - 139, 2016.

[25] Z. Li, Z. Duan, and F. L. Lewis, "Distributed robust consensus control of multi-agent systems with heterogeneous matching uncertainties," Automatica, vol. 50, no. 3, pp. 883 - 889, 2014.

[26] J. Mei, W. Ren, and J. Chen, "Distributed consensus of second-order multi-agent systems with heterogeneous unknown inertias and control gains under a directed graph," IEEE Transactions on Automatic Control, vol. 61, no. 8, pp. 2019-2034, 2016.

[27] Z. Feng, G. Hu, W. Ren, W. E. Dixon, and J. Mei, "Distributed coordination of multiple unknown euler-lagrange systems," IEEE Transactions on Control of Network Systems, vol. PP, no. 99, pp. 1-1, 2016.

[28] G. S. Seyboth, D. V. Dimarogonas, K. H. Johansson, P. Frasca, and F. Allgwer, "On robust synchronization of heterogeneous linear multiagent systems with static couplings," Automatica, vol. 53, pp. 392 - 399, 2015.

[29] J. Mei, W. Ren, B. Li, and G. Ma, "Distributed containment control for multiple unknown second-order nonlinear systems with application to networked lagrangian systems," IEEE Transactions on Neural Networks and Learning Systems, vol. 26, no. 9, pp. 1885-1899, 2015.

[30] S. Ghapani, J. Mei, W. Ren, and Y. Song, "Fully distributed flocking with a moving leader for lagrange networks with parametric uncertainties," Automatica, vol. 67, pp. $67-76,2016$.

[31] T. Olds, "The mathematics of breaking away and chasing in cycling," European Journal of Applied Physiology, vol. 77, no. 6, pp. 492-497, 1998.

[32] J. Stout, "Rules of the bunch - etiquette on the bike," Ride, Cycling review, vol. 63, no. 1, 2014.

[33] D. Jia, K. Lu, J. Wang, X. Zhang, and X. Shen, "A survey on platoon-based vehicular cyber-physical systems," IEEE Communications Surveys Tutorials, vol. 18, no. 1, pp. 263-284, 2016.

[34] R. Kianfar, M. Ali, P. Falcone, and J. Fredriksson, "Combined longitudinal and lateral control design for string stable vehicle platooning within a designated lane," in 17th International IEEE Conference on Intelligent Transportation Systems (ITSC), 2014, pp. 1003-1008.

[35] G. Tao, "Multivariable adaptive control: A survey," Automatica, vol. 50, no. 11, pp. 2737 - 2764, 2014.

[36] - Adaptive Control Design and Analysis. Wiley, 2003.

[37] P. Ioannou and J. Sun, Robust Adaptive Control. Dover Publications, 2012.

[38] H. K. Khalil, Nonlinear Systems (3rd Edition). Pearson, 2001.

[39] B. van Arem, C. J. G. van Driel, and R. Visser, "The impact of cooperative adaptive cruise control on traffic-flow characteristics," IEEE Transactions on Intelligent Transportation Systems, vol. 7, no. 4, pp. 429-436, 2006.

[40] J. Ploeg, E. Semsar-Kazerooni, G. Lijster, N. van de Wouw, and H. Nijmeijer, "Graceful degradation of cooperative adaptive cruise control," IEEE Transactions on Intelligent Transportation Systems, vol. 16, no. 1, pp. 488-497, 2015.

[41] K. C. Dey, L. Yan, X. Wang, Y. Wang, H. Shen, M. Chowdhury, L. Yu, C. Qiu, and V. Soundararaj, "A review of communication, driver characteristics, and controls aspects of cooperative adaptive cruise control (cacc)," IEEE Transactions on Intelligent Transportation Systems, vol. 17 , no. 2 , pp. 491-509, 2016. 
[42] X. Guo, J. Wang, F. Liao, and R. S. H. Teo, "String stability of heterogeneous leader-following vehicle platoons based on constant spacing policy," in IEEE Intelligent Vehicles Symposium (IV), Gothenburg, Sweden, 2016, pp. 761-766.

[43] Y. Abou Harfouch, S. Yuan, and S. Baldi, "An adaptive approach to cooperative longitudinal platooning of heterogeneous vehicles with communication losses," in 20th IFAC World Congress, July 9th-14th, Toulouse, France, 2017.

[44] G. Lymperopoulos and P. Ioannou, "Adaptive control of networked distributed systems with unknown interconnections," in 2016 IEEE 55th Conference on Decision and Control (CDC), 2016, pp. 3456-3461.

[45] Q. Sang and G. Tao, "Adaptive control of piecewise linear systems: the state tracking case," IEEE Transactions on Automatic Control, vol. 57, no. 2, pp. 522-528, 2012.

[46] S. Yuan, B. D. Schutter, and S. Baldi, "Adaptive asymptotic tracking control of uncertain time-driven switched linear systems," IEEE Transactions on Automatic Control, 2017, in press.

[47] W. Wang, C. Wen, J. Huang, and Z. Li, "Hierarchical decomposition based consensus tracking for uncertain interconnected systems via distributed adaptive output feedback control," IEEE Transactions on Automatic Control, vol. 61, no. 7, pp. 1938-1945, 2016. 